\title{
Semi-infinite metallic system: QST versus DFT
}

\author{
Kostrobij P. P., Markovych B. M., Ryzha I. A. \\ Lviv Polytechnic National University, \\ 12 S. Bandera Str., 79013, Lviv, Ukraine
}

(Received 3 August 2021; Revised 20 December 2021; Accepted 5 January 2022)

\begin{abstract}
Modeling and investigation of thermodynamic characteristics of spatially-finite metallic systems is an essential task of modern nanophysics. We show that the widely used DFT (density functional theory) is less efficient than the QST (quantum-statistical theory) approach.
\end{abstract}

Keywords: semi-infinite metal, density functional theory, many-particle density matrix. 2010 MSC: $82 \mathrm{~B} 24$

DOI: $10.23939 / \mathrm{mmc} 2022.01 .178$

\section{Introduction}

The modern development of nanotechnology has led to the necessity of constructing and studying mathematical models of spatially-inhomogeneous systems. The most straightforward of such systems are the spatially-finite metallic systems, for the study of which the density functional theory (DFT) is widely used. This theory was proposed by Hohenberg and Kohn in the papers $[1,2]$ and is essentially a further development of the Thomas-Fermi theory [3], well-known in the theory of multielectron atoms. However, the DFT is, in fact, a one-particle method and does not allow to take into account the multiparticle correlations correctly $[4,5]$. It is particularly true when considering the discreteness of ionic subsystem [6-8].

An alternative to DFT is the quantum-statistical theory (QST), proposed in $[9,10]$, which is a generalization of the reference system approach known in the study of multielectron systems [11].

In this paper the results of DFT and QST for calculating the thermodynamic characteristics of the semi-infinite metallic systems are compared. The effectiveness of QST is shown.

\section{Mathematical model of semi-infinite metallic systems}

Advances in the theoretical description of the properties of metals with a surface are associated with the usage of a simple model for such a system, which was proposed in the works $[9,10]$.

We consider a semi-infinite metallic system whose point ions have a charge $\mathcal{Z} e$ and the Cartesian coordinates $\mathbf{R}_{j}=\left(X_{j}, Y_{j}, Z_{j}\right)$. Coordinates $\left(X_{j}, Y_{j}\right) \in \mathbb{R}^{2}, Z_{j} \leqslant 0, z=0$ are the equation of a "metal-vacuum" separation surface (plane) $, j=1, \ldots, N_{\text {ion }}, e$ is the electron charge. Free electrons of a semi-infinite metal have coordinates $\mathbf{r}_{i}, i=1, \ldots, N$.

The Hamiltonian of this model can be presented as follows:

$$
H=-\frac{\hbar^{2}}{2 m} \sum_{i=1}^{N} \Delta_{i}+\frac{1}{2} \sum_{i \neq j=1}^{N} \frac{e^{2}}{\left|\mathbf{r}_{i}-\mathbf{r}_{j}\right|}+\sum_{j=1}^{N_{\text {ion }}} \frac{\left(\mathbf{P}_{j}, \mathbf{P}_{j}\right)}{2 M}+\frac{1}{2} \sum_{j \neq k=1}^{N_{\text {ion }}} \frac{(\mathcal{Z} e)^{2}}{\left|\mathbf{r}_{j}-\mathbf{r}_{k}\right|}+\sum_{i=1}^{N} \sum_{j=1}^{N_{\text {ion }}} w\left(\mathbf{r}_{i}, \mathbf{R}_{j}\right)
$$

where the first term is the kinetic energy of free electrons, the second term is the Coulomb interaction energy between free electrons, the third term is the kinetic energy of ions ( $\mathbf{P}$ is the ion momentum operator), the fourth term is the potential energy of the inter-ionic interaction, and the last term is the energy of electron-ion interaction [13]; $\hbar$ is the Planck constant, $m$ and $M$ are the electron mass and ion mass, respectively. 
We assume that, in general, the system is electroneutral, i.e. the following condition is met

$$
\mathcal{Z} N_{\text {ion }}=N \text {. }
$$

It is convenient to present the potential $w\left(\mathbf{r}_{i}, \mathbf{R}_{j}\right)$ of electron-ion interaction in this form

$$
w\left(\mathbf{r}_{i}, \mathbf{R}_{j}\right)=w\left(\left|\mathbf{r}_{i}-\mathbf{R}_{j}\right|\right)+\Delta w\left(\mathbf{r}_{i}, \mathbf{R}_{j}\right),
$$

where $w\left(\left|\mathbf{r}_{i}-\mathbf{R}_{j}\right|\right)$ is the periodic potential of electron-ion interaction in the case of infinite metal (pseudopotential [11]), $\Delta w\left(\mathbf{r}_{i}, \mathbf{R}_{j}\right)$ is the deviation of electron-ion interaction potential in a semiinfinite metal from the periodic potential $w\left(\left|\mathbf{r}_{i}-\mathbf{R}_{j}\right|\right)$.

We extract from Hamiltonian (1) the Hamiltonian of the semi-infinite jellium model $H_{\text {jell }}$ [11] (reference system). We have (see [11])

where

$$
H=H_{\text {jell }}+\tilde{H}_{\mathrm{ii}}+\sum_{i=1}^{N} \sum_{j=1}^{N_{\text {ion }}} \delta w\left(\left|\mathbf{r}_{i}-\mathbf{R}_{j}\right|\right)
$$

and

$$
\begin{gathered}
\tilde{H}_{\mathrm{ii}}=\sum_{j=1}^{N_{\text {ion }}} \frac{\mathbf{P}_{j}^{2}}{2 M}+\frac{1}{2} \sum_{j \neq k=1}^{N_{\text {ion }}} \frac{(\mathcal{Z} e)^{2}}{\left|\mathbf{R}_{j}-\mathbf{R}_{k}\right|}-\frac{1}{2} \int_{V} \mathrm{~d} \mathbf{R} \int_{V} \mathrm{~d} \mathbf{R}^{\prime} \frac{\rho_{\text {jell }}(\mathbf{R}) \rho_{\text {jell }}\left(\mathbf{R}^{\prime}\right)}{\left|\mathbf{R}-\mathbf{R}^{\prime}\right|} \\
H_{\text {jell }}=H_{\text {jell }}^{\text {unif }}+\sum_{i=1}^{N} V_{\text {surf }}\left(\mathbf{r}_{i}\right)+\frac{1}{2} \int_{V} \mathrm{~d} \mathbf{R} \int_{V} \mathrm{~d} \mathbf{R}^{\prime} \frac{\rho_{\text {jell }}(\mathbf{R}) \rho_{\text {jell }}\left(\mathbf{R}^{\prime}\right)-\left(\frac{e N}{V}\right)^{2}}{\left|\mathbf{R}-\mathbf{R}^{\prime}\right|},
\end{gathered}
$$

$$
H_{\text {jell }}^{\text {unif }}=-\frac{\hbar}{2 m} \sum_{i=1}^{N} \Delta_{i}+\frac{1}{2} \sum_{i \neq j=1}^{N} \frac{e^{2}}{\left|\mathbf{r}_{i}-\mathbf{r}_{j}\right|}-\frac{e^{2} N}{V} \sum_{i=1}^{N} \int_{V} \mathrm{~d} \mathbf{R} \frac{1}{\left|\mathbf{r}_{i}-\mathbf{R}\right|}+\frac{(e N)^{2}}{2 V^{2}} \int_{V} \mathrm{~d} \mathbf{R} \int_{V} \mathrm{~d} \mathbf{R}^{\prime} \frac{1}{\left|\mathbf{R}-\mathbf{R}^{\prime}\right|}
$$

is the Hamiltonian of homogeneous jellium (see [11]);

$$
V_{\text {surf }}\left(\mathbf{r}_{i}\right)=V_{\text {jell }}\left(\mathbf{r}_{i}\right)+V_{\text {ion }}\left(\mathbf{r}_{i}\right),
$$

the surface potential acting on electrons;

$$
\begin{gathered}
V_{\text {jell }}\left(\mathbf{r}_{i}\right)=e \int_{V} \mathrm{~d} \mathbf{R} \frac{\frac{e N}{V}-\rho_{\text {jell }}(\mathbf{R})}{\left|\mathbf{r}_{i}-\mathbf{R}\right|}, \quad V_{\text {ion }}\left(\mathbf{r}_{i}\right)=\sum_{j=1}^{N_{\text {ion }}} \Delta w\left(\mathbf{r}_{i}, \mathbf{R}_{j}\right) \\
\delta w\left(\left|\mathbf{r}_{i}-\mathbf{R}_{j}\right|\right)=w\left(\left|\mathbf{r}_{i}-\mathbf{R}_{j}\right|\right)+\frac{1}{N_{\text {ion }}} \int_{V} \mathrm{~d} \mathbf{R} \frac{e \rho_{\text {jell }}(\mathbf{R})}{\left|\mathbf{r}_{i}-\mathbf{R}\right|}
\end{gathered}
$$

is the difference potential - the difference between the ion pseudopotential and the electrostatic potential of the positive charge of semi-infinite jellium.

In $(4)-(7)$

$$
\rho_{\text {jell }}(\mathbf{R})=\frac{e N}{V} \theta(-Z)
$$

is the ion density distribution in the semi-infinite jellium model, $V=S L / 2$ is the volume of a semiinfinite metal, $S$ is the surface area of a semi-infinite metal, $L$ is the range of change of electron $z$-coordinate normal to the surface: $z \in(-L / 2 ; L / 2)$. Henceforth, we assume that $S \rightarrow \infty, L \rightarrow \infty$, $\theta(x)$ is the Heaviside step function.

The grand partition function $\Xi$ of the proposed model for semi-infinite metal

$$
\Xi=\operatorname{Sp} \exp \{-\beta(H-\mu N)\}
$$

in the adiabatic approximation [13] can be presented as follows:

$$
\Xi=\exp \left\{-\beta \tilde{H}_{\mathrm{ii}}\right\} \operatorname{Sp} \exp \left\{-\beta\left(H_{\text {jell }}-\mu N-\tilde{V}_{\text {ei }}\right)\right\} \equiv \Xi_{1} \cdot \Xi_{2},
$$

where

$$
\Xi_{1}=\exp \left\{-\beta \tilde{H}_{\mathrm{ii}}\right\}
$$


and

$$
\Xi_{2}=\exp \left\{-\beta\left(H_{\text {jell }}-\mu N-\tilde{V}_{\text {ei }}\right)\right\}
$$

is the grand partition function for the electronic subsystem of a semi-infinite metal in the field of fixed ions. In (11), (13) $\mu$ is the chemical potential, and $\beta$ is thermodynamic beta [11].

Calculating $\Xi_{2}(13)$ or thermodynamic potential $\Omega_{2}=-\frac{1}{\beta} \ln \Xi_{2}$ [11] is an essential task to obtain various thermodynamic and structural characteristics of metals with a separation surface.

\section{DFT}

The basis of DFT approach for calculating $\Omega_{2}$ are theorems of Hohenberg-Kohn [1] and Mermin [15], which state that the thermodynamic potential $\Omega$ can be presented as follows:

$$
\Omega[n(\mathbf{r})]=F[n(\mathbf{r})]+\int v(\mathbf{r}) n(\mathbf{r}) \mathrm{d} \mathbf{r} .
$$

The minimum $\Omega$ determines the electron density $n(\mathbf{r})$, unambiguously connected with an external potential $v(\mathbf{r})$. The external potential is the potential created by the discrete ionic subsystem, i.e.

$$
v(\mathbf{r})=\sum_{j=1}^{N_{\text {ion }}} w\left(\mathbf{R}_{j}-\mathbf{r}\right) .
$$

Here $w(\mathbf{R}-\mathbf{r})$ is the pseudopotential of electron interaction $[6,7]$.

In $(14)$

$$
F[n]=\operatorname{Sp}\left\{\left(T+U+\frac{1}{\beta} \ln \hat{\rho}_{0}\right) \hat{\rho}_{0}\right\}
$$

where

$$
\hat{\rho}_{0}=\frac{\exp \{-\beta(T+U-\mu N)\}}{\operatorname{Sp} \exp \{-\beta(T+U-\mu N)\}}
$$

is the operator of equilibrium distribution, obtained from the condition for minimum (14), and the electron density

$$
n(\mathbf{r})=\operatorname{Sp}\left(\hat{\rho}_{0} \hat{n}(\mathbf{r})\right) .
$$

$\hat{n}(\mathbf{r})$ is the electron density operator, $T$ is the electron kinetic energy operator $T=-\frac{\hbar^{2}}{2 m} \sum_{i=1}^{N} \Delta_{i}$, and $U$ is the operator of potential energy of interaction $U=\frac{1}{2} \sum_{i \neq j=1}^{N} \frac{e^{2}}{\left|\mathbf{r}_{i}-\mathbf{r}_{j}\right|}$.

Since condition $\beta \mu \gg 1$ [11] is always satisfied, $\Omega+\mu N=E$ is the energy of the system, and therefore the minimization condition

$$
E_{\min }=\min _{n(\mathbf{r})} E[n(\mathbf{r})]
$$

gives the Euler-Lagrange equation [1], namely

$$
\delta E[n(\mathbf{r})]=\int \delta n(\mathbf{r})\left\{\frac{\delta}{\delta n(\mathbf{r})} T[n(\mathbf{r})]+v_{\mathrm{eff}}(\mathbf{r})-\mu\right\}=0,
$$

where

$$
v_{\text {eff }}(\mathbf{r}) \equiv v(\mathbf{r})+\int \frac{n\left(\mathbf{r}^{\prime}\right)}{\left|\mathbf{r}-\mathbf{r}^{\prime}\right|} \mathrm{d} \mathbf{r}^{\prime}+\frac{\delta}{\delta n(\mathbf{r})} E_{\mathrm{xc}}[n(\mathbf{r})] .
$$

The condition for minimum (16) leads to solving a system of one-particle Schrödinger equations

$$
\left(-\frac{\hbar^{2}}{2 m} \Delta_{i}+v_{\text {eff }}(\mathbf{r})-\varepsilon_{i}\right) \psi_{i}(\mathbf{r})=0
$$

taking into account the following relations

$$
n(\mathbf{r})=\sum_{j=1}^{N(\mu)}\left|\psi_{j}(\mathbf{r})\right|^{2}
$$




$$
\Delta v_{\text {eff }}(\mathbf{r})=-4 \pi n(\mathbf{r})
$$

Summation in (19) runs over states $j$ such that $\varepsilon_{j} \leqslant \mu$.

The system of equations (18)-(20) is solved by numerical methods. The main problem is constructing the functional $E_{\mathrm{xc}}[n(\mathbf{r})][1,2]$, for which various approximations are used (see [3]). But all currently available approximations lead to accurate results only in two extreme cases: (1) the density $n(\mathbf{r})$ changes very slowly $(\nabla n(\mathbf{r}) \rightarrow 0) ;(2) n(\mathbf{r}) \gg 1$ (when the kinetic energy operator $T$ can be neglected). Moreover, the chemical potential $\mu$ is uncertain.

\section{QST}

In QST, proposed in [9-11], the calculation of the grand statistical sum $\Xi_{2}$ reduces to the calculation of many-particle density matrices, and in the case of local pseudopotentials the energy $E$ of the considered model can be represented as follows:

$$
E=E_{0}+\delta E^{(1)}+\delta E^{(2)}+\ldots,
$$

where

$$
\begin{aligned}
E_{0}= & \varepsilon_{0}+\left.\frac{1}{2} \frac{N S}{V} \int_{-L / 2}^{L / 2} \mathrm{~d} z F_{1}^{0}(z) \int_{0}^{1} \mathrm{~d} \lambda\left(g\left(\mathbf{r}_{\|}, z, z, \lambda\right)-\frac{e^{2}}{\left|\mathbf{r}_{\|}\right|}\right)\right|_{\mathbf{r}_{\|}=0} \\
& +\frac{1}{2} \frac{N^{2} S}{V^{2}} \int_{S} \mathrm{~d} \mathbf{r}_{\|} \int_{-L / 2}^{L / 2} \mathrm{~d} z_{1} \int_{-L / 2}^{L / 2} \mathrm{~d} z_{2}\left(F_{2}^{0}\left(\mathbf{r}_{\|}, z_{1}, z_{2}\right)-F_{1}^{0}\left(z_{1}\right) F_{1}^{0}\left(z_{2}\right)\right) \int_{0}^{1} \mathrm{~d} \lambda g\left(\mathbf{r}_{\|}, z_{1}, z_{2}, \lambda\right),
\end{aligned}
$$

and the screened potential of inter-electronic interaction

$$
g\left(\mathbf{r}_{\|}, z_{1}, z_{2}, \lambda\right)=\frac{1}{S} \sum_{\mathbf{q}} \exp \left\{\mathrm{i}\left(\mathbf{q}, \mathbf{r}_{\|}\right)\right\} g\left(\mathbf{q}, z_{1}, z_{2}, \lambda\right)
$$

is the solution of integral equation

$$
g\left(\mathbf{q}, z_{1}, z_{2}, \lambda\right)=\nu\left(\mathbf{q}, z_{1}-z_{2}\right)+\frac{\beta}{S L^{2}} \lambda \int_{-L / 2}^{L / 2} \mathrm{~d} z \int_{-L / 2}^{L / 2} \mathrm{~d} z^{\prime} \nu\left(\mathbf{q}, z_{1}-z^{\prime}\right) D\left(\mathbf{q}, z^{\prime}, z\right) g\left(\mathbf{q}, z_{1}, z_{2}, \lambda\right) .
$$

Here $D$ is the "density-density" correlation function of the noninteracting system of electrons in the field of "surface potential" $V_{\text {surf }}(\mathbf{r})$ [11],

$$
\begin{aligned}
\nu(\mathbf{q}, z) & =\frac{1}{L} \sum_{k} \frac{4 \pi e^{2}}{q^{2}+k^{2}} \exp (-\mathrm{i} k z), \\
\varepsilon_{0}= & -\frac{1}{\beta} \sum_{\mathbf{k}_{\mid,}, \alpha} \ln \left[1+\exp \left\{\beta\left(\mu-E_{\alpha}\left(\mathbf{k}_{||}\right)\right)\right\}\right]
\end{aligned}
$$

is the total energy of the system of noninteracting electrons in the field of "surface potential" $V_{\text {surf }}(\mathbf{r})$, $E_{\alpha}\left(\mathbf{k}_{\|}\right)$are the one-particle electron energies (see [11]).

In the case of homogeneity of a semi-infinite metal in a plane parallel to the separation plane

$$
\begin{gathered}
\delta E^{(1)}=S \sum_{j=1}^{N_{\text {ion }}} \int \mathrm{d} z\langle\delta w\rangle_{p}\left(z-Z_{j}\right) n(z), \\
\delta E^{(2)}=-\beta \frac{N(N-1)}{2 V^{2}} \sum_{i=1}^{N_{\text {ion }}} \sum_{j=1}^{N_{\text {ion }}} \int \mathrm{d} r_{1} \int \mathrm{d} r_{2} \delta w\left(\left|\mathbf{r}_{1}-\mathbf{R}_{j}\right|\right) \delta w\left(\left|\mathbf{r}_{2}-\mathbf{R}_{i}\right|\right) F_{2}\left(\mathbf{r}_{1}, \mathbf{r}_{2}\right) \\
+\beta \frac{N}{2 V} \sum_{j=1}^{N_{\text {ion }}} \sum_{i=1}^{N_{\text {ion }}} \int \mathrm{d} r \delta w\left(\left|\mathbf{r}-\mathbf{R}_{j}\right|\right) \delta w\left(\left|\mathbf{r}-\mathbf{R}_{i}\right|\right) F_{1}(z)
\end{gathered}
$$

Mathematical Modeling and Computing, Vol. 9, No. 1, pp. 178-185 (2022) 


$$
+\beta \frac{N^{2}}{2 V^{2}} S^{2} \sum_{j=1}^{N_{\text {ion }}} \int \mathrm{d} z_{1}\langle\delta w\rangle_{p}\left(z_{1}-Z_{j}\right) F_{1}\left(z_{1}\right) \sum_{i=1}^{N_{\text {ion }}} \int \mathrm{d} z_{2}\langle\delta w\rangle_{p}\left(z_{2}-Z_{i}\right) F_{1}\left(z_{2}\right) .
$$

In (22)-(28) $F_{1}(\mathbf{r})$ and $F_{2}\left(\mathbf{r}_{1}, \mathbf{r}_{2}\right)$ are the unary and binary Bogolyubov distribution functions, respectively [16], which are also the functionals of chemical potential $\mu ; n(z)=\frac{N}{V} F_{1}(z)$ is the electron density distribution;

$$
\langle\delta w\rangle_{p}\left(z-Z_{i}\right)=\frac{1}{S} \int \mathrm{d} \mathbf{r}_{\|} \delta w\left(\sqrt{r_{\|}^{2}+\left(z-Z_{i}\right)^{2}}\right) ;
$$

$F_{1}^{0}\left(\mathbf{r}_{2}\right)$ and $F_{2}^{0}\left(\mathbf{r}_{1}, \mathbf{r}_{2}\right)$ are respectively the unary and binary distribution functions of the system of noninteracting electrons in the field of "surface potential" $V_{\text {surf }}\left(\mathbf{r}_{i}\right)$, defined by formula (7) (see [9-11]). The general scheme for obtaining expressions for $\delta E^{(n)}$ is given in [11].

Knowing the expression for $\Omega$, one can get the chemical potential $\mu$ from the condition

$$
N=\langle N\rangle=-\frac{\partial \Omega}{\partial \mu}
$$

Thus, in contrast to DFT, expressions (22)-(29) state a clear dependence of the total energy $E$ of the electronic subsystem of a semi-infinite metal on the distribution functions and show that just knowledge of the unary (density distribution) function is not enough to calculate the energy of a semi-infinite metallic system.

\section{Some numerical results and conclusions}

In order to perform numerical calculations of the thermodynamic characteristics (energy, density of particle distribution, etc.) in QST, it is first necessary to know the chemical potential $\mu$ of the electronic subsystem, determined by equation (29). It is essential to note it is necessary to correctly take into account the electroneutrality condition, as shown in Refs. $[17,18]$.

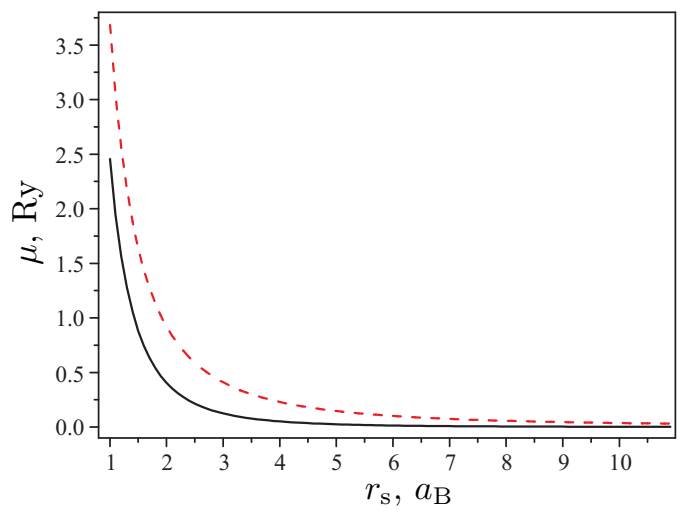

Fig. 1. Chemical potential $\mu$ for electronic subsystem of semi-infinite metal.

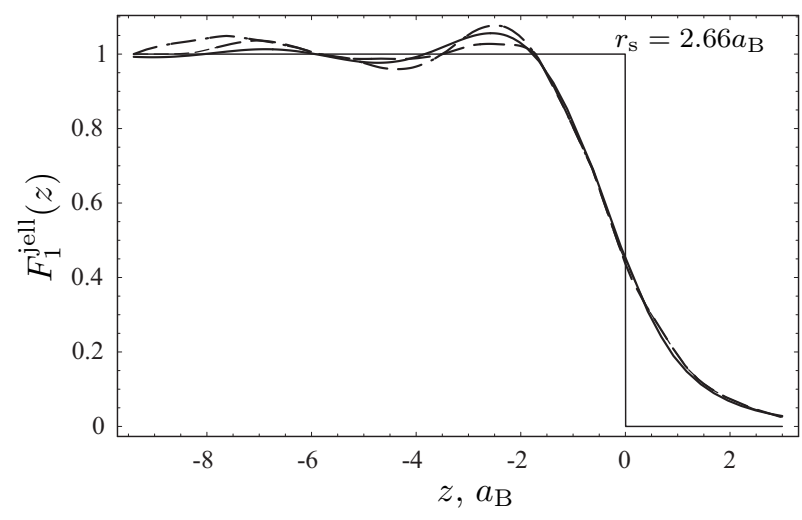

Fig. 2. Unary electron distribution function $F_{1}(z)$. Solid line - QST calculation, dashed line - using the Monte Carlo method, dash-dot - DFT calculation.

Fig. 1 shows the results of calculating the chemical potential $\mu$ as a function of the Gell-MannBrueckner parameter $r_{\mathrm{s}}$ [11]. As can be seen from the calculated results, depending on the electron concentration, the chemical potential $\mu$ behaves in the same way as for the unlimited metal; that is, the presence of the separation surface has little effect on its behavior.

Fig. 2 presents the results of calculating the unary distribution function $F_{1}(z)$ for the values of parameter $r_{\mathrm{s}}=2.66$ and comparing them with the results obtained in the DFT approach and the Monte Carlo method calculations. 
As can be seen from Fig. 2, the results of calculating $F_{1}(z)$ in the near-surface region agree better with the Monte Carlo method calculations [12] than the results of the DFT approach.

The results of binary distribution function $F_{2}\left(\mathbf{r}_{\|}, z_{1}, z_{2}\right)$ calculation for the case, when one of the electrons is located at the point $\left(r_{\|}=0, z_{2}=-6 a_{\mathrm{B}}\right)$ and $\left(r_{\|}=0, z_{2}=-4 a_{\mathrm{B}}\right)$, are given in Fig. 3 . The results of such calculations in DFT are impossible.

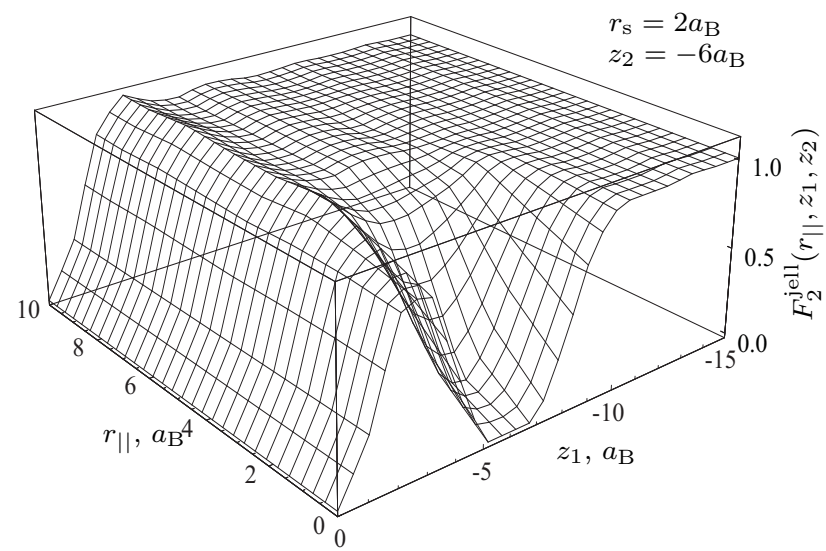

$a$

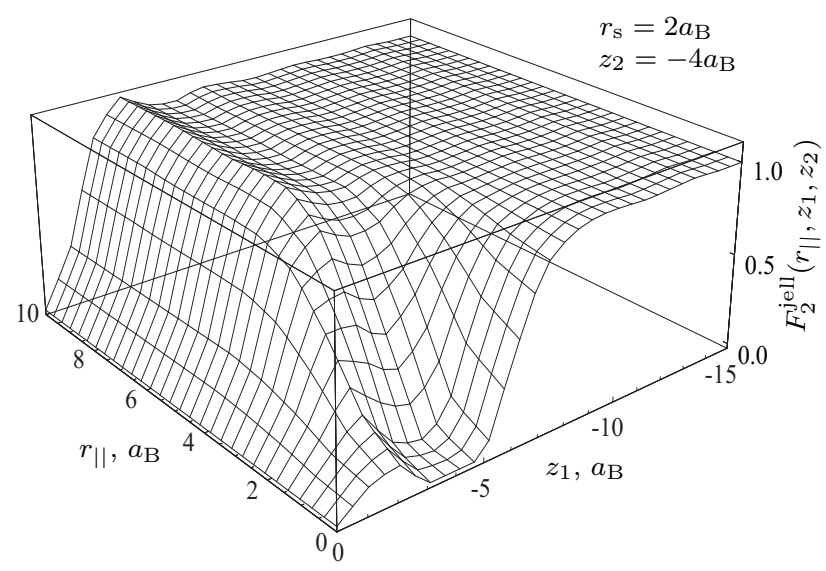

$c$

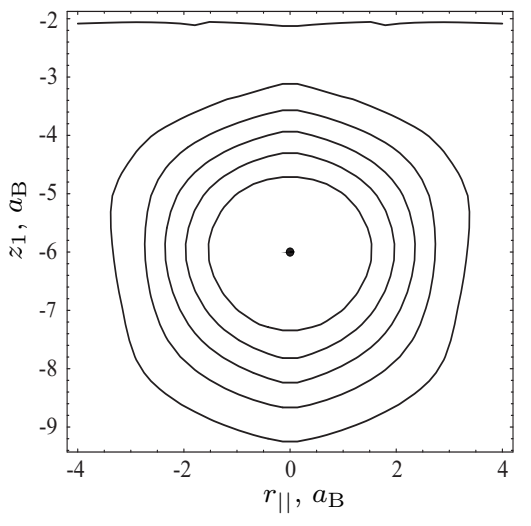

$b$

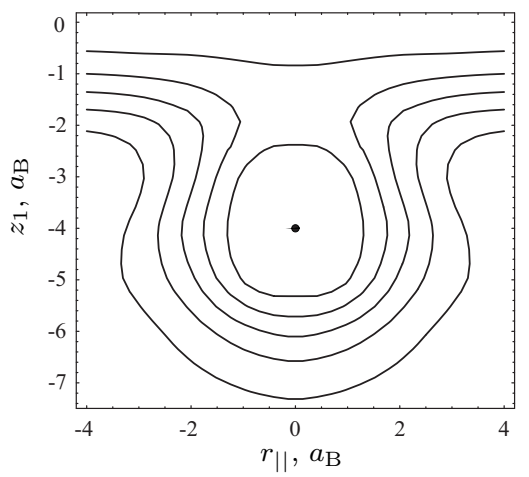

$d$

Fig. 3. Binary electron distribution function $F_{2}\left(\mathbf{r}_{\|}, z_{1}, z_{2}\right)$ and its projection on $\left(r_{\|}, z_{1}\right)$-plane for $z_{2}=-6 a_{\mathrm{B}}$ (top) and $z_{2}=-4 a_{\mathrm{B}}$ (bottom).

Fig. 4 shows the results of calculating the surface energy $\sigma$ (defined according to Kohn-Lang [2]) as a function of concentration. As shown in Fig. 4, the surface energy values calculated in the QST approach agree much better with the experimental data for simple metals than those obtained in DFT. Moreover, the surface energy $\sigma$ in QST is positively defined (unlike DFT) in the whole range of electron concentrations. As the electron concentrations decrease (as the $r_{\mathrm{s}}$ parameter increases), the QST values of $\sigma$ start to agree with the DFT cal-

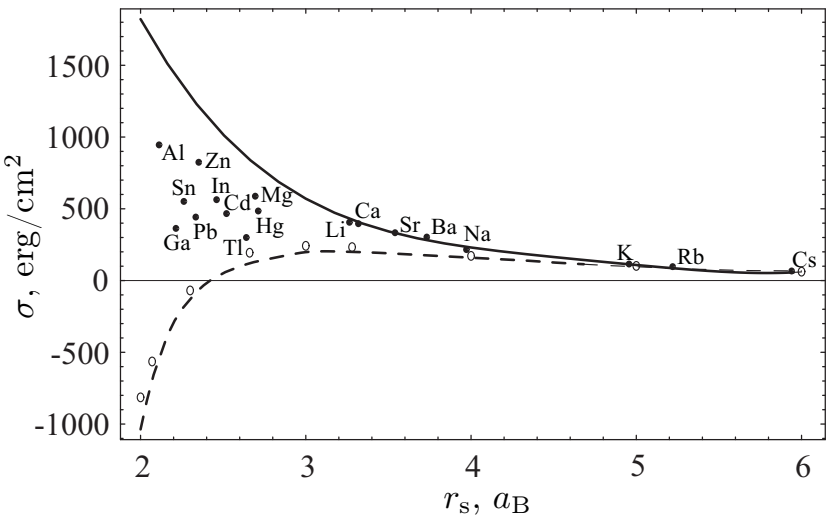

Fig. 4. Surface energy $\sigma$. culations. This behavior of DFT calculations of $\sigma$ indicates the fundamental problems with correct consideration of correlation effects in DFT for metallic systems with a separation surface. 
Very interesting are the results of calculating the effective potential $g\left(\mathbf{r}_{\|}, z_{1}, z_{2}\right)$ of inter-electronic interaction, which cannot be performed in DFT. These calculations are presented in Fig. 5.
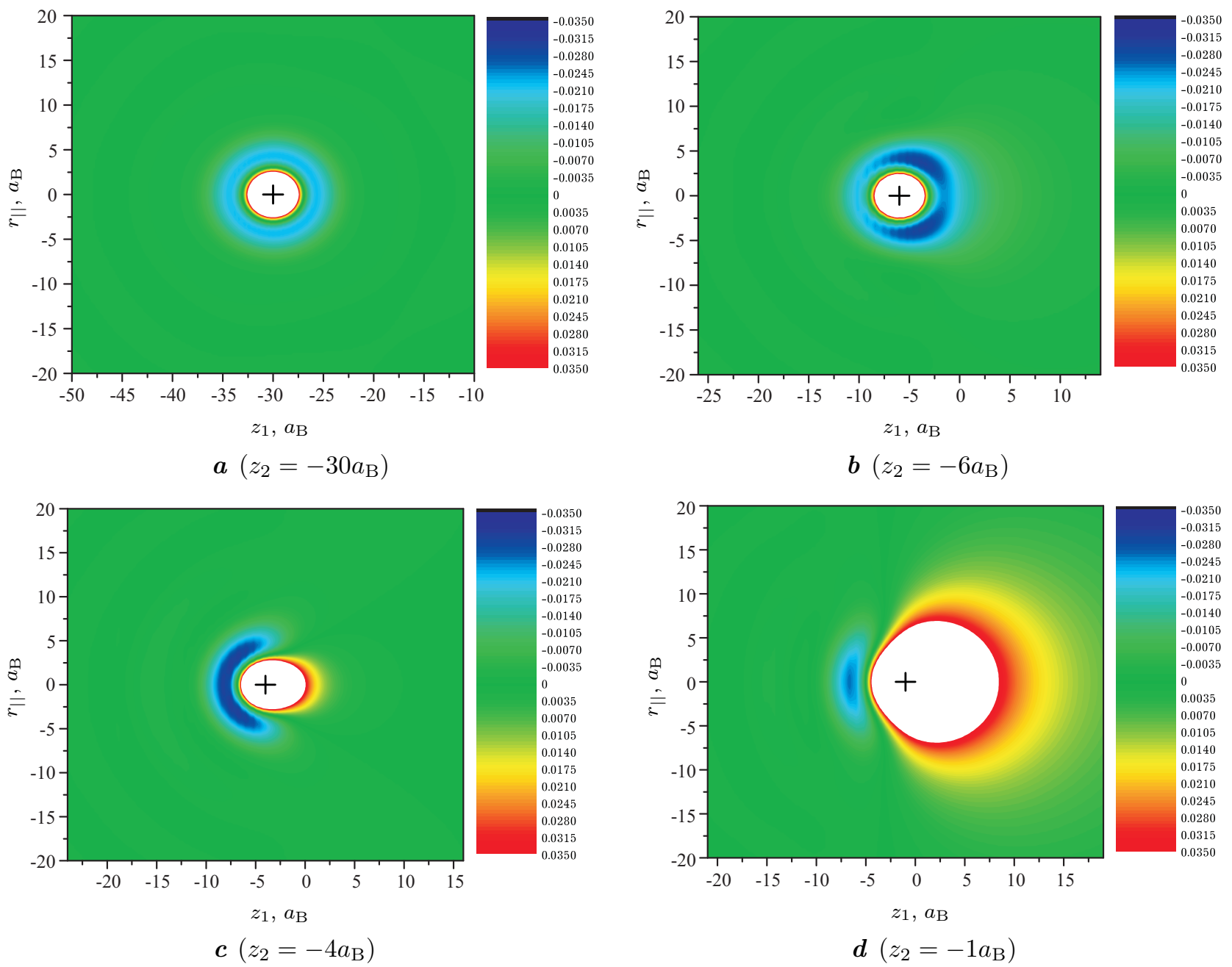

Fig. 5. Dependence of the effective potential of inter-electronic interaction, taking into account the correction for the local field (the Hubbard approximation), on the distance between electrons in the separation plane and the normal coordinate of one of the electrons, the other coordinate is fixed. Calculations performed for $r_{\mathrm{s}}=4.86 a_{\mathrm{B}}$.

The results of calculation show that in the depth of metal the effective potential of the interelectronic interaction is axially symmetric; this symmetry is lost when approaching the separation plane.

The obtained results suggest that QST is more effective for studying spatially-finite metallic systems than DFT. The use of expression (21) for calculating the total energy of spatially-finite metallic systems allows, if one knows the distribution functions $F_{1}(z), F_{2}\left(z_{1}, z_{2}\right)$ (see (19)-(28)) of the reference frame (electrons in the field of surface potential), to calculate the thermodynamic characteristics of such systems.

[1] Hohenberg P., Kohn W. Inhomogeneous electron gas. Physical Review. 136 (3B), B864-B871 (1964).

[2] Lang N. D., Kohn W. Theory of metal surfaces: Charge density and surface energy. Physical Review B. 1 (12), 4555-4568 (1970).

[3] Theory of the Inhomogeneous Electron Gas. Edited by Lundqvist S. and March N. H. Springer, Boston, MA (1983). 
[4] Mattsson A. E., Kohn W. An energy functional for surfaces. The Journal of Chemical Physics. 115 (8), 3441-3443 (2001).

[5] Eguiluz A. G., Heinrichsmeier M., Fleszar A., Hanke W. First-principles evaluation of the surface barrier for a Kohn-Sham electron at a metal surface. Physical Review Letters. 68 (9), 1359-1362 (1993).

[6] Fiolhais C., Henriques C., Sarría I., Pitarke J. M. Metallic slabs: Perturbative treatments based on jellium. Progress In Surface Science. 67 (1-8), 285-298 (2001).

[7] Dobson J. F., Rose J. H. Surface properties of simple metals via inhomogeneous linear electronic response. I. Theory. Journal of Physics C: Solid State Physics. 15 (36), 7429-7456 (1982).

[8] Eguiluz A. G. Lattice relaxation at an aluminum surface: Self-consistent linear-electronic-response approach. Physical Review B. 35 (11), 5473-5486 (1987).

[9] Kostrobij P. P., Markovych B. M. Semi-infinite metal: Perturbative treatment based on semi-infinite jellium. Condensed Matter Physics. 11 (4), 641-651 (2008).

[10] Kostrobij P. P., Markovych B. M. Semi-infinite jellium: Thermodynamic potential, chemical potential, and surface energy. Physical Review B. 92 (7), 075441 (2015).

[11] Vavrukh M. V., Kostrobij P. P., Markovych B. M. Basis approach in the theory of multielectron systems. Rastr-7, Lviv (2017), (in Ukrainian).

[12] Acioli P. H., Ceperley D. M. Diffusion Monte Carlo study of jellium surfaces: Electronic densities and pair correlation functions. Physical Review B. 54 (23), 17199-17207 (1996).

[13] Vakarchuk I. O. Quantum mechanics. Ivan Franko National University of Lviv, Lviv (2012), (in Ukrainian).

[14] Abrikosov A. A., Gorkov L. P., Dzyaloshinskii I. E. Methods of quantum field theory in statistical physics. Fizmatgiz, Moscow (1962), (in Russian).

[15] Mermin N. D. Thermal Properties of the Inhomogeneous Electron Gas. Physical Review. 137 (5A), A1441-A1443 (1965).

[16] Bogolyubov N. N. Selected works on statistical physics. Moscow University Press, Moscow (1979), (in Russian).

[17] Kostrobij P. P., Markovych B. M., Polovyi V. Y. Influence of the electroneutrality of a metal layer on the plasmon spectrum in dielectric-metal-dielectric structures. Mathematical Modeling and Computing. 6 (2), 297-303 (2019).

[18] Kostrobij P. P., Markovych B. M. Effect of Coulomb interaction on chemical potential of metal film. Philosophical Magazine. 98 (21), 1991-2002 (2018).

\title{
Напівобмежена металева система: QST проти DFT
}

\author{
Костробій П. П., Маркович Б. М., Рижа І. А.
}

Національний університет "Львівська політехніка", вул. С. Бандери, 12, Лъвів, 79013, Україна

\begin{abstract}
Розглянуто два підходи до моделювання просторово-обмежених металевих систем: DFT та QST. В обох підходах енергія напівобмежених металів подається у вигляді ряду за степенями псевдопотенціалу електрон-іонної взаємодії. Однак QST-підхід, на відміну від DFT-підходу, дозволяє коректно врахувати обмінно-кореляційні ефекти електронної підсистеми.
\end{abstract}

Ключові слова: напівобмежений метал, теорія функиіоналу густини, багаточастинкова матриця густини. 